\title{
Improve the Quality of Service in Time Division Multiple Access based Vehicular Ad Hoc Network
}

\author{
Monisha Gupta \\ Assistant Professor, Department of CSE \\ Arunachal University of Studies \\ Namsai, India
}

\author{
Dr. Sahil Verma \\ Associate Professor, Department of CSE \\ Lovely Professional University \\ Jalandhar, India
}

\begin{abstract}
Vehicular Ad-Hoc Network (VANET) is an emerging wireless technologies brought by the latest advances in mobile communication. It is now gaining attention and momentum in the automobile industry. Vehicles need to equip with wireless transceivers and control modules to communicate with each other. A range of few hundred meters is the limited range up to which the vehicle can communicate with other vehicles. Therefore, the messages hop using several nodes in order to provide end-to-end communication across huge distances. To transfer messages between the nodes, efficient and reliable dispatch of packets is required. In addition to reliable delivery, messages should be exchanged without any collision. These challenges can be satisfied with fair, reliable and highly efficient Medium Access Control (MAC) protocols. It is difficult to implement the medium control protocols in VANET environment due to mobility of nodes at very high level. It is important to allocate the time-slots between vehicles in case of these protocols. Arbitrating the access of wireless channels is the major objective of MAC protocol. A channel assignment policy scheme is proposed which improves performance of the network as well as reduces the increased collision in the network. The proposed scheme is executed in NS2 and outcomes are analyzed with respect to certain criterion. It is evaluated that proposed procedure performs better than other parameters when compared.
\end{abstract}

Keywords - Vehicular ad hoc networks; QoS; wireless; MAC

\section{INTRODUCTION}

Vehicular Ad-Hoc Network is one of the types of Mobile AdHoc Networks where nodes are vehicles or road-side units. The nodes are highly mobile with constrained movements, sufficient energy and computing power and hybrid network architecture. For communication, vehicles should be equipped with wireless transceivers and computerized control modules. Roadside Units (RSUs) are permanent network nodes that act as gateways to the Internet or servers to access any relevant information. This exchange of information is a building block for Intelligent Transportation System (ITS).

VANETs are mainly designed to provide safety on roads, improve traffic management conditions and on-board entertainment. Vehicles can collect the essential information and share it with other vehicles. This information can be related to the traffic jam situation, road condition detection, accident warning, tourism information, etc. The collected information would be helpful for the users to plan their route. It acts as a safety aid for the driver and passengers too. If the person caught up with some abnormal situation, current positional information of the vehicle can be sent to the police station or nearby hospital. In recent years, VANET has gained much popularity due to its contribution towards effective solution for dealing with traffic related problems and saving the lives of pedestrians, drivers and passengers on the road. Each vehicle acts like a wireless node which can connect within 100 to 300 meters range for exchanging data. As a result, a mobile network of extensive range is formed, wherein nodes keep dropping out of the network as they move out of the signal range. Due to this high mobility, VANET are vulnerable to erratic physical threats that trigger probable vulnerabilities for promising attackers.

In VANET, communication exists either between the vehicles or between vehicle and road-side units called as Vehicle-toVehicle communication (V2V) and Vehicle-to-Road Infrastructure communication (V2I) respectively. $\mathrm{V} 2 \mathrm{~V}$ is completely ad-hoc based communication in contrast to V2I which is infrastructure based communication.

\section{A. Characteristics}

The distinct features of VANETs make MANET architectures and protocols difficult to be employed in VANET. However, the following points highlight some of the basic characteristics of vehicular ad-hoc networks which make them dissimilar from other mobile ad-hoc network listed as follows:-

- High mobility- Unlike other ad-hoc networks, nodes of VANET are highly mobile and they show variation in speed according to different environment. Therefore, the protocols implemented in VANET must adapt to dynamically changing topology because of nodes mobility.

- No Energy Constraint- Nodes are vehicles, so it has ample amount of energy (power) and computation power.

- Wide range of Quality of Service RequirementsVANET provides three fundamental services such as- Realtime applications, traffic related applications and useroriented applications. All these applications differ from each other in terms of variable Quality of Service requirements.

- Mobility Model- While designing a network protocol for VANETs, the mobility model and prediction about the future position of a vehicle are important. Communication environment is either a highway or a city traffic scenario. Movement of vehicles in VANET can be predictable to some amount as the vehicles' movements restricted by the road topology.

\section{B. Challenges}

Due to various VANET characteristics as described above, there exist many challenging research issues [12] on which several researchers, academicians and industry people are working. Their effort result in positive solutions for some issues but still there are some issues which need more attention for the successful deployment of VANETs. All these challenging issues are summarized as follows: - 
- Dynamically varying network topology- As the nodes are highly mobile in VANET, the MAC protocol designed need to be seamlessly adapt to frequent changes in network topology.

- Different QoS requirements- To satisfy the distinct applications like safety or non-safety applications of VANETs, there is a need of modifications in the spectrum. Existing IEEE standards are not sufficient for higher bandwidth requirements of VANETs. A dynamic protocol designed with cross layered architecture rather than pure layered architecture can better help in fulfilling these requirements.

- Security- It directly conveys the fact that this network lack efficient defense mechanism. The security techniques ideally need to be implemented on-demand and that they should be able to deal with big dynamic clusters at any point in time. Maintaining security and privacy in VANET is a big challenge for the researchers.

- Hidden-terminal problem- Another challenge is hidden terminal problem which hinders the performance of VANET. It cannot be solved using RTS/CTS control messages for broadcast messages.

- No central coordination- As VANET is an infrastructure less network so it doesn't have a central coordinator. The MAC protocols developed for VANET must distribute the control among the vehicles and they must exchange control messages to remove collisions. For V2R communication, RSUs (infrastructure) is required at the intersection of roads or junctions. Deployment of RSUs is a challenging task. Always deploy the RSUs in an optimal manner such that maximum area would be covered with less number of RSUs.

- $\quad$ High speed- To handle frequent network disruptions and vehicles high mobility, scalable algorithms are needed. In a city environment, the network is very dense, whereas in highways or rural areas, vehicle density is very low. According to the environment or road types, vehicles change its speed. Scalable and robust protocols are needed for handling the issues of each layer.

- Simulation- Before deploying the protocols in a real environment, it requires extensive rigorous testing. Simulation is the most feasible choice for testing and simulating a protocol's behaviour. However, reproducing realistic vehicular motion on a simulated environment is another challenging issue on which several researchers have been working.

- Socio-Economic- It is very difficult to convince the early-adopters to install VANET equipment in their vehicles. Various techniques were tried ranging from law enforcement, attractive deployment applications, etc. But the infrastructure on the road-side, IT-management and backhaul connectivity issues arises which affected many road operators, communities, etc.

- Authentication- The trust based security protocol and authentication mechanism for the vehicles need a careful design strategy.

\section{Applications}

VANETs are primarily developed to provide safety and management of traffic by reducing the number of accidents in the highway and urban areas. Applications of VANET are mainly classified into three categories which are described below: -

- Safety applications- It comprises a number of scenarios where the valuable lives of the citizens are of prime concern. This can avoid the accident and save the lives of a number of people. It even inform all the nearby vehicles on the roads by sending alert messages to clear the path for emergency vehicle and even turn on all the lights to green the moment the vehicle comes at the traffic signal. This application alerts vehicles moving closer to a specific zone with specific driving requirements

- Traffic Management and Monitoring applications- They are the tools for making our transportation system intelligent. An optimal route along the way can be provided to the user. These applications provide real time traffic view so the user can decide the best path as per different constraints like less congested, a shorter one, etc. The roadside units can get knowledge about the problem in any route and broadcast this information to the surrounding vehicles to avoid these routes. Parking Availability Notification (PAN) helps vehicles find available space in the parking lot of a certain geographical area.

- Non-safety applications- It incorporate information, entertainment, traffic management and monitoring applications. An information and entertainment application cover comfort of the passenger and the driver. A driver can get any information beforehand such as enquiring about a nearby petrol station or restaurant or parking slot availability in an area.

To lessen the number of accidents and save people from accidents, automotive industries are focusing to make the ITS a successful innovation. Automotive industries main motive is to provide safety to the passengers. Various automotive industries have started a joint collaboration by forming an organization called as Car2Car Communication Consortium (C2CCC) which aims in providing reliable traffic related messages in inter-vehicle communication. Figure 1 depicts the various applications of VANETs.

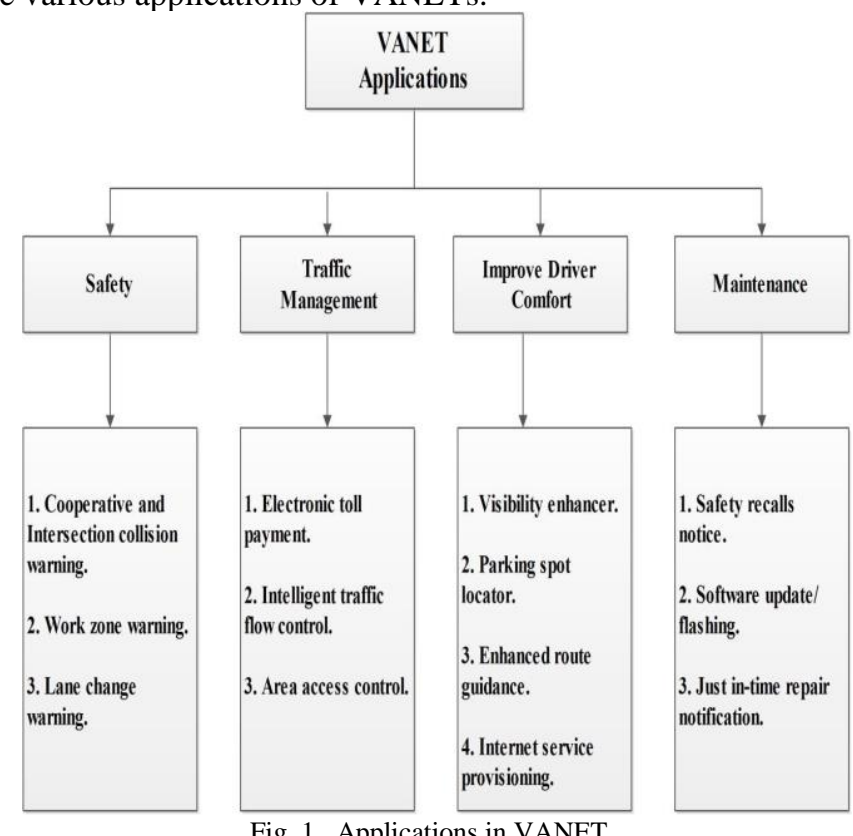




\section{RELATED WORK}

Various researches were done to reduce the collision and improve the quality of service which aims in increasing the packet delivery ratio and throughput of the network. Various Time Division Multiple Access (TDMA) based MAC protocols have been proposed as it provides bounded delay with less packet loss ratio than other multiple access schemes and experiences no interference from concurrent transmission.

In [1], the author proposed a new MAC protocol named as Triggered CCHI Multichannel MAC protocol. This protocol considered vehicles to be equipped with On-board Unit providing wireless communication using IEEE 802.11p/WAVE 1609.4 standards. The author also proposed a virtual TDMA algorithm to exchange status messages between neighboring nodes. TCM-MAC reduces the packet loss ratio than CAVI-MAC (Context-Aware Variable Interval MAC) by the transmission of CMR (Cluster Member Request) or CHR (Cluster Head Request) packets efficiently which transfer the safety packets to the destination without any collision. These packets also remove the hidden terminal problem. It reduces the delay of safety messages by successfully switching the SCHI and CCHI. In intra-cluster communication, throughput is indirectly proportional to vehicle density and directly proportional to vehicle density as clusters will be very far away from each other to receive clusters messages. But the protocol overhead is highest in TCM-MAC due to transfer of additional control packets. As a result, TCM-MAC has a better performance metrics than CAVI-M.

Dynamic Broadcast Storm Mitigation Algorithm (DBSMA) is proposed in [2]. In VANET, all the vehicles are equipped with wireless technology to exchange information between them. The main purpose of WAVE (Wireless Access in Vehicular Environment) is to develop protocols to enable vehicle-to-vehicle communication so that accident is reduced on roads. To analyze the congestion in network, Distributed Congestion Control (DCC) mechanism was proposed. But the DCC mechanism failed in higher node density which affects the vehicular network. In such situations, DBSMA approach is used. When there is traffic jam, DBSMA reduces the broadcast delay to reduce the congestion problem. Before reaching any obstacle the vehicles are informed prior to their arrival of the spot which makes the vehicles slow down and increases the vehicular density at the spot. When density increases, a large number of messages are broadcasted but the speed is slow so network resources are rarely exhausted. Performance of DBSMA is measured by the Channel Busy Ratio (CBR) metric. CBR is used to measure the channel resources availability and utilization as handshaking mechanism is absent in Vehicular Network. Simulation result proved that DBSMA is better than DCC in both density and mobility conditions. DBSMA provide $130 \%$ efficiency against DCC under the effect of broadcast storm. It is easily implemented and computed.

Md. Kowsar Hossain, et.al, proposed a new distributed MAC protocol called as ResVMAC [3] which is an improved version of RR-ALOHA and MARR-ALOHA. Medium Access Control is necessary for any wireless medium communication. Using MAC, all the vehicles sense the shared wireless channel to transfer the data without any collisions. TDMA based MAC protocols in VANET are basically of two types:- 1)Protocols following the DSRC standard. For eg- VeMAC, TC-MAC, HER-MAC. 2)Protocols that do not follow the DSRC standard. For egRR-ALOHA, MARR-ALOHA. In RR-ALOHA reservation of Basic Channel (BCH) is done through sending of REQ packets which contains the request for accessing the channel. If channel is free then one-hop neighbor will send the FI packet to the sender. MARR-ALOHA was designed to mitigate the limitations of RR-ALOHA i.e. reduction of collision caused due to REQ and FI packets. ResVMAC frame structure is same as that of RR-ALOHA dividing time slots into virtual frames and respective frames are further divided into fixed number of BCHs. REQ packet helps vehicle to send data by reserving a free $\mathrm{BCH}$. After reservation of channel, the sender broadcast the packet until it receives negative acknowledgement from its one-hop neighbors. Due to hidden terminal problem collision of REQ packets occurs, but size of REQ packet is small so it ensures that this happens in small probability. It is necessary for every node in the VANET to have a communication link with its neighbor nodes. In this context, role of NACK (Negative Acknowledgement) exists. If a sender request to access a $\mathrm{BCH}$ by sending REQ packet; and it is already occupied by some other neighbor node then the node upon receiving the REQ packet will send NACK packet to sender which indicates the sender that the $\mathrm{BCH}$ is already occupied.

$\mathrm{Du}$, et.al, provided solution for a major issue in VANET i.e. collision of packets in a high density network caused due to accessing of a single slot by more than one node. The solution is a TDMA based protocol known as PTMAC [4] which can identify the collision and reduce it effectively. Earlier proposals were made to reduce collisions but they were not effective for unbalanced traffic in both the directions and also for four-way intersections, but it was the first protocol designed to remove all such problems. While comparing this proposed protocol with earlier protocols like ADHOC MAC, etc, it results in fewer collisions, packet delivery rate is also higher and even in the presence of large traffic its performance is not degraded.

In [5], the author proposed a Cluster based TDMA system (CBT) for efficient communication between inter vehicles. The main motive is to maintain a reliable and collision free communication between intra clusters and inter cluster. It elects a VANET Coordinator (VC). This protocol assumed that vehicles are installed with GPS system and all the vehicles are synchronized using this system. The author failed to discuss the situation how the slots are released and allocated when vehicles move or when a vehicle comes inside a cluster.

A multichannel MAC protocol combining CSMA with TDMA and SDMA is presented [6] to solve hidden/exposed terminal problem and a broadcast service which is reliable as well as ratio between $\mathrm{SCH}$ and $\mathrm{CCH}$ channels are dynamically adjusted according to density of nodes. Here, a comparison between the performance of CS-TDMA and IEEE standard as well as with the SOFT MAC and VeMAC is done. While using CS-TDMA, the nodes can easily access the control channel; packet delivery is fast in highly 
bidirectional dense network which is slow in other protocols. It also addressed switching of channel problem concurrently. The utilization of wireless resources is done efficiently and provides guaranteed QoS for safety messages. But the throughput decreases rapidly when the traffic increases so it is limited to medium dense network.

Hang Yu, et.al, focuses on improving the performance of STDMA. STDMA is particularly designed to be used for real-time communication [7]. It provided guaranteed delivery even in the highly density network. It focuses on a scenario where a highly dynamic topology exists and each node must communicate with every node, and then determine the performance of STDMA using the simulation results.

In [8], the author proposed an Adaptive Collision Free MAC (ACFM) protocol which is a centralized protocol that assigns slots to vehicles dynamically. It ensures that the nodes make efficient utilization of the slots. The control messages which is transmitted by road-side units consists of synchronization information as well as slot reservation information.

H. Omar, et.al, outlined multichannel protocol named as VeMAC [9]. It is developed to remove the limitation of ADHOC MAC, a single channel protocol not suitable to be used for DSRC architecture. This protocol has certain preliminaries which need to be followed by the node. This paper focus on the working of VeMAC, provided an qualitative comparison regarding the performance of VeMAC and ADHOC MAC in highway and city scenarios using MATLAB simulation results.

In [10], the author proposed an Adaptive Real-Time Distributed MAC protocol which functions similar to that of ADHOC protocol. It is mainly designed to combat the limitation of the latter protocol. The results of the simulation are analyzed and the protocol performs well even in case of large number of nodes. It can easily adapt to the frame length by creating less overhead.

Risk-Aware Dynamic MAC protocol is an upgrade version of ACFM for Vehicular Cooperative Collision Avoidance (CCA) system [11]. In this protocol, a frame is subdivided into two subparts- the first part is for delivering beacon messages and the second part is for delivering warning messages. Secondly, an algorithm is also designed to determine the average number of collisions due to the vehicles. The protocol is considered as a reliable and efficient one. It has surpass the conventional protocol i.e. IEEE 802.11 p protocol in terms of criteria like packet delivery ratio and transmission delay.

\section{PROPOSED METHODOLOGY}

Neighbouring vehicles contend to get a free slot in a time frame. A node when occupies a slot tries to get the same slot in the subsequent frame until collision occurred. A node transmits beacon messages which includes occupied slot information and neighbour node information which helps in reducing merging and access collision. In general process is furnished in four stages: 1) Transfer of beacon messages. 2) Predicting the probable collision. 3) Reporting of collision to neighbour. 4) Allotment of time-slot. The structure of beacon message is illustrated in the below figure 2 . The first slot is always reserved to send collision warning messages.
Reservation of slot depends on the speed of vehicles and Id. The speed of colliding nodes is calculated and the one with highest speed acquires the new slot or the node with lowest id acquires it. One of the collided nodes is used to send the warning message of collision. It creates a list of neighbour that is within two hop of each other and their respective allocated slot information. The beacon message is exchanged to help the neigbour node to know about the slot allocated to each node. In VANET, two types of collision occur namely access collision and merging collision. These collisions mostly occur while using a distributed scheme to access a time slot by the vehicles. When two vehicles belonging from two different two

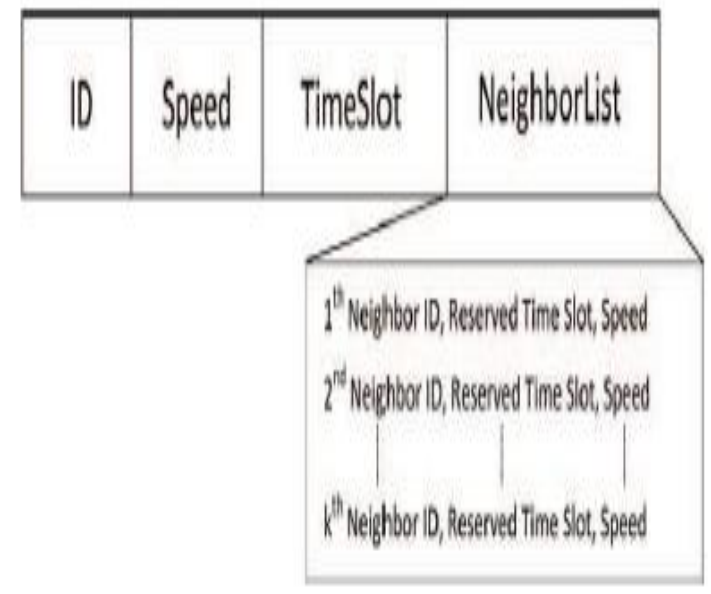

Fig. 2. Beacon message format

hop neighbourhood uses the same slot but due to the speed they seems to change their position and become members of the same two-hop neighbourhood thereby leading to merging collision.

\section{A. Predicting the merging collision}

Let there be 5 nodes respectively as $\mathrm{N}_{\mathrm{A}}, \mathrm{N}_{\mathrm{B}}, \mathrm{N}_{\mathrm{C}}, \mathrm{N}_{\mathrm{D}}$ and $\mathrm{N}_{\mathrm{E}}$ which reserved slots as 3, 6, 8, 4 and 3. At $t_{1}$ time interval, $N_{B}$ use slot 6 and $\mathrm{N}_{\mathrm{A}}$ use slot 3 and both are in communication range of each other whereas $\mathrm{N}_{C}, \mathrm{~N}_{\mathrm{D}}$ and $\mathrm{N}_{\mathrm{E}}$ are in communication range. There will be no collision in $t_{1}$ as $N_{A}$ and $N_{E}$ both are far away from each other. But at time $t_{2}, N_{B}$ move towards $\mathrm{N}_{\mathrm{C}}$ and become neighbours and due to the issue of beaconing message, it sees that $\mathrm{N}_{\mathrm{A}}$ and $\mathrm{N}_{\mathrm{E}}$ uses same time slot and may become neighbour in near future which increase the probability of merging collision. This probability leads to the issue of warning message by nodes $\mathrm{N}_{B}$ and $\mathrm{N}_{C}$ The whole scenario is depicted in the figure 4.

The warning message of collision need to be transmitted without any collision. So, to ensure that first time slot of a frame is always reserved to transmit warning message. The warning message includes information such as name of nodes which will undergo collision, its reserved time slot and its two hop neighborhood. Every vehicle knows about the average speed of its neighbors with the help of beacon message as beacon message include the average speed of the entire neighbor. The neighbor of the node which is selected to acquire the new slot after collision is the one which issues a warning message of collision in the first time slot. After the reception of collision message, the nodes checks the identity 
of nodes which will undergo collision and the resultant node stops transmitting data on the required slot and tries to change the slot.

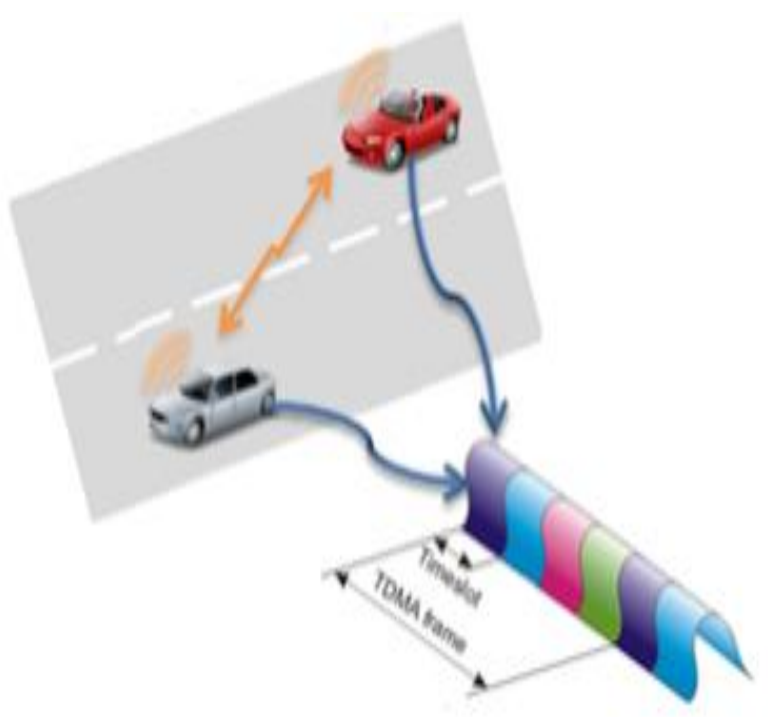

Fig. 3. Access Collision
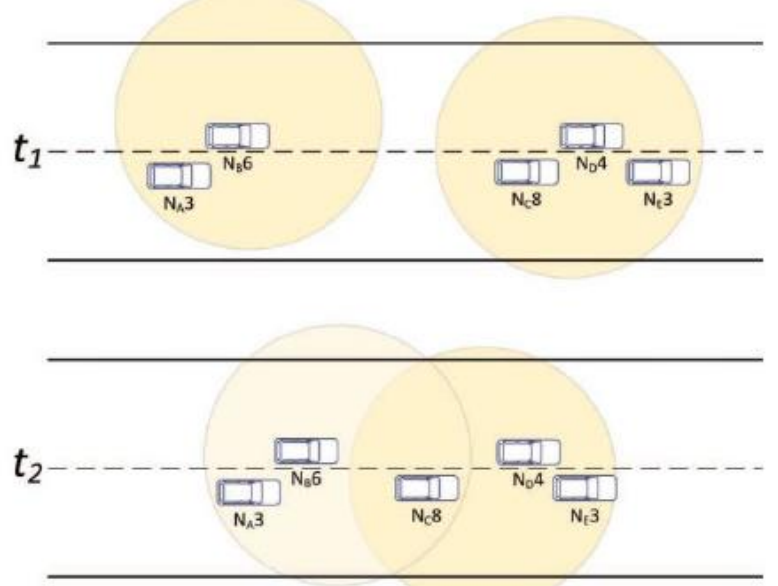

Fig. 4. Merging collision within 2-hop neighbourhood

\section{B. Efficient Time slot algorithm}

1. Let $\mathrm{V}_{1}$ and $\mathrm{V}_{2}$ be two collided nodes

2. Compare and compute vehicles with high speed $\mathrm{S}_{1}$ and $\mathrm{S}_{2}$.

3. If $\mathrm{S}_{1}>\mathrm{S}_{2}$

then $S_{1}$ will acquire the time slot first.

Else If $\mathrm{S}_{1}<\mathrm{S}_{2}$

then $S_{2}$ will acquire the time slot.

4. Else

Nodes with lowest Id will acquire the time slot.

End If

5. If $\mathrm{V}_{1}$ and $\mathrm{V}_{2}$ are in neighbour list, then

A list of 2-hop neighbour is created

Provide collision message to 2-hop neighbour

Include nodes $\mathrm{V}_{1}$ and $\mathrm{V}_{2}$ in the collision message.

End If.

\section{RESULTS AND DISCUSSION}

We have associated this research with channel access in vehicular ad hoc network. The novel protocol designed to assign wireless channel efficiently. The simulation of the proposed model is performed in network simulator version 2 by considering traffic simulator SUMO. Highway scenario is used for the simulation with average vehicle speed as 100 $\mathrm{km} / \mathrm{hr}$. Other simulation parameters are listed in the table 1 . The performance is tested in terms of packet delivery ratio, throughput, access and merging collision.

Table I. Simulation Parameters

\begin{tabular}{|c|c|}
\hline Parameters & Values \\
\hline Area for simulation & Highway \\
\hline Length of Road & $2 \mathrm{Km}$ \\
\hline Simulation Time & $60 \mathrm{~s}$ \\
\hline Vehicle Densities & $60-120$ Vehicle/Km \\
\hline Transmission Range & $200,250,300,350$ \\
\hline Avg. Vehicle Speed & $100 \mathrm{~km} / \mathrm{hr}$ \\
\hline Time Frame Length & $100 \mathrm{~ms}$ \\
\hline Time Slot Duration & $1 \mathrm{~ms}$ \\
\hline
\end{tabular}

The proposed work is compared with existing protocols like A-ADHOCMAC and R-MAC with respect to certain performance parameters like throughput, packet delivery ratio, access collision and merging collision. The throughput should generally be high in order to deliver the packets in large amount in short duration of time. Figure 5 depicts that network throughput is increased when the channel access policy is implemented in the network. The throughput of the two scenarios are compared in which channel access policy is implemented and second scenario in which no channel access policy is implemented. The graph clearly depicts the throughput value of the proposed protocol and existing protocol. The throughput value is highest in the proposed protocol i.e. 10 when number of nodes is 6 as compared to other protocols with values as 5 and 7 of A-ADHOCMAC and R-MAC. Even after increment of the number of nodes, the throughput value is not affected; it yields the highest value i.e. 27 with number of nodes as 22 when compared to other protocols A-ADHOCMAC and R-MAC the value is 20 and 23 respectively.

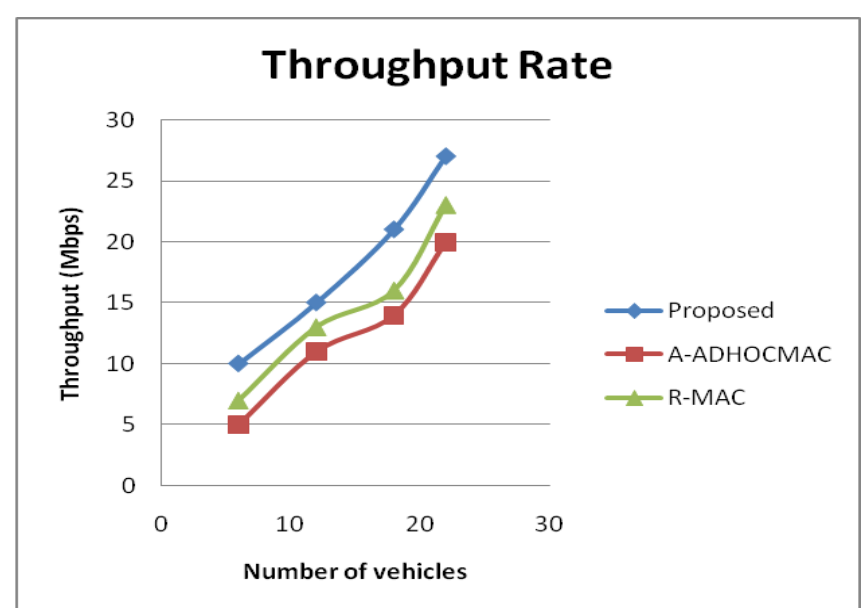

Fig. 5. Throughput Comparison with channel assignment policy and without channel assignment policy 
Packet Delivery ratio becomes an integral parameter to measure the efficiency of the proposed work. It needs to be greater in every protocol which means that packets that are sent by the sender are being received by the receiver correctly. Figure 6 depicts that packet delivery ratio with channel assignment policy is high as compared to without channel assignment. As per the results obtained, the packet delivery rate of proposed protocol is high as compared to other existing protocols like A-ADHOCMAC and R-MAC. For example: when nodes are 6 in number, the rate of packet delivery is highest in proposed protocol i.e. 0.95 as compared to A-ADHOCMAC and R-MAC with 0.8 and 0.9. Even after the increase of nodes with 22 in number, the proposed protocol outperforms existing one. It has been recorded that proposed protocol gives 0.85 and A-ADHOCMAC gives 0.68 and R-MAC gives 0.78 respectively.

Access collision need to be reduced to increase the delivery of data packets. In Figure 7 access collision comparison is done between with and without channel assignment policy. It is analyzed that collision is lower in channel assignment policy as compared to without channel assignment policy. According to the results, when vehicle density is 58, the number of access collision is higher in A-AHOCMAC than R-MAC and proposed protocol i.e. 11 and 8 respectively as compared to that of 13 . As the vehicle density increases, the number keeps increasing. It becomes highest with the value of 39 in A-AHOCMAC and lowest in the proposed protocol i.e. 28 .

Merging collision must be avoided as if not lowered then after every collision slot allocation procedure need to be done resulting in delay of sending the packets by the sender. Figure 8 depicts that merging collision comparison is done between with and without channel assignment policy. It is analyzed that collision is lower in channel assignment policy as compared to without channel assignment policy. According to the results, when vehicle density is 58, the number of merging collision is higher in A-AHOCMAC than R-MAC and proposed protocol i.e. 7 and 3 respectively as compared to that of 11. As the vehi-

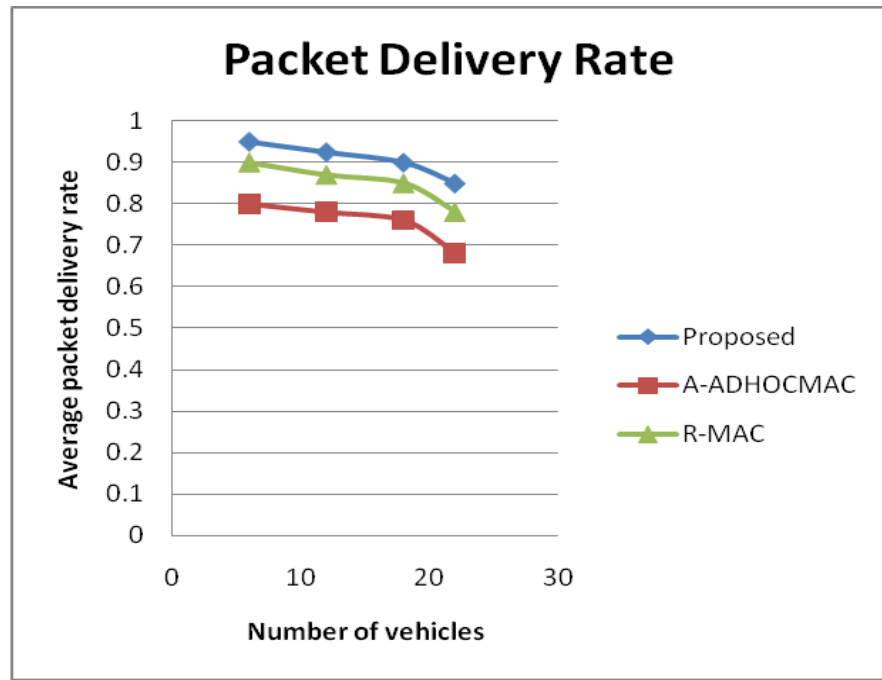

Fig. 6. Packet Delivery Ratio Comparison with channel assignment policy and without channel assignment policy -cle density increases, the number keeps increasing. It becomes highest with the value of 30 in A-AHOCMAC and lowest in the proposed protocol i.e. 16.

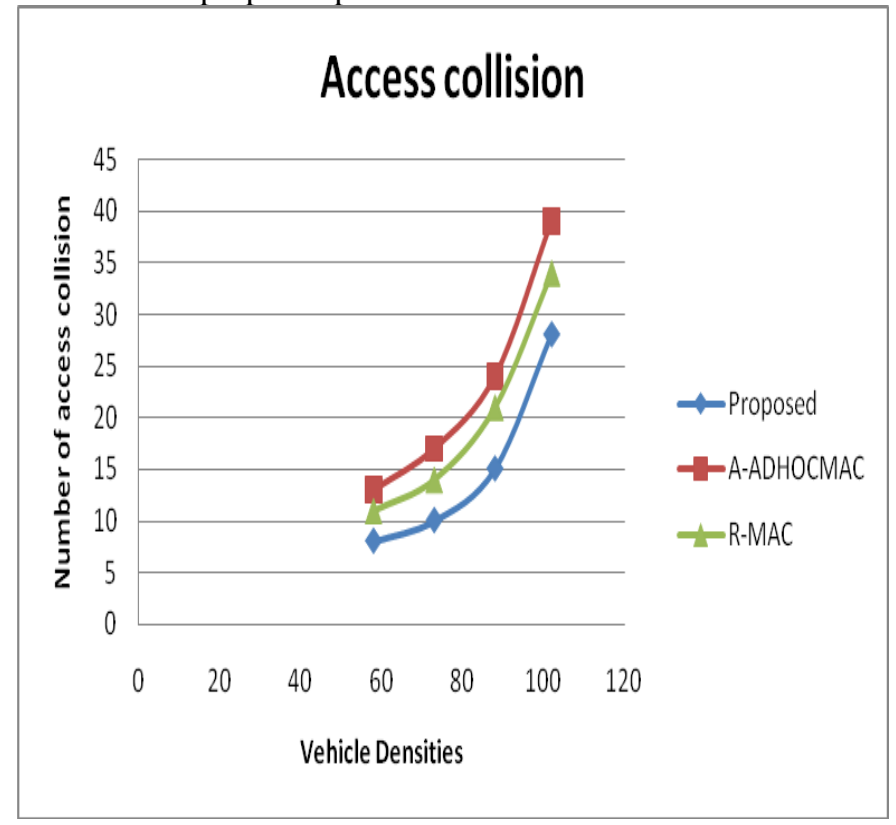

Fig. 7. Access Collision Comparison with respect to channel assignment policy and without channel assignment policy

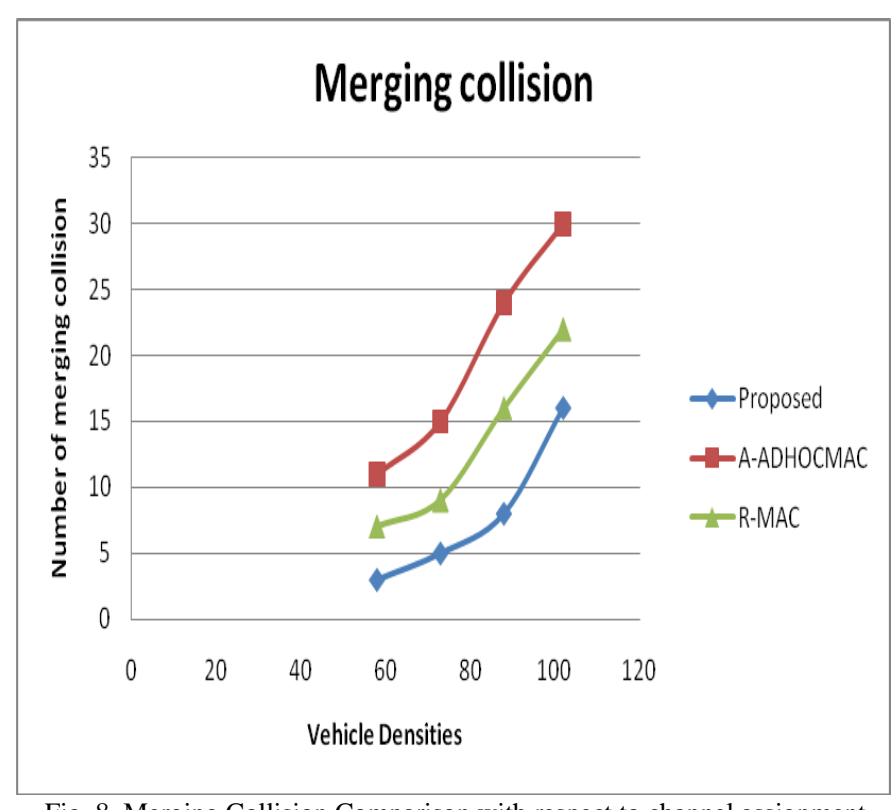

Fig. 8. Merging Collision Comparison with respect to channel assignment policy and without channel assignment policy

\section{CONCLUSION AND FUTURE WORK}

It is concluded that vehicular ad hoc network has dynamically varying topology due to which channel assignment become serious issues of the system. The proposed scheme for VANET is implemented in network simulator version 2 . The collision is detected among vehicles that are within two-hop neighbourhood. The results are analyzed in terms of certain parameters like throughput, packet delivery ratio, access and merging collision. It is analyzed that throughput is high in proposed work as compared to without channel assignment policy. The packet delivery ratio of the 
without channel assignment is low as compared to with channel assignment policy. It is analyzed that both access as well as merging collision is drastically reduced when contrasted with existing without channel assignment plan. Accessing the medium with different nodes containing diverse information will lead to collision and variable delay. On account of this variable delay and indefinite collision, a novel backoff method is important to determine each of these issues in future. To lessen interference between covering territories, few protocols make utilization of different access procedures like CDMA, FDMA, etc. which makes them complex protocol. Settling these issues will require more prominent endeavors in the near future. In spite of the impressive research pointing to enhance the execution of MAC protocols in VANETs, no perfect solution has yet been identified that can meet the QoS requirement at the MAC layer and resolve every one of the issues caused by the unique attributes of VANETs.

\section{REFERENCES}

[1] R. Pal, A. Prakash, and R. Tripathi, "Triggered CCHI Multichannel MAC protocol for Vehicular Ad Hoc Networks," Vehicular Communications, vol. 12, pp. 14-22, 2018.

[2] E. A. Feukeu and T. Zuva, "Dynamic Broadcast Storm Mitigation Approach for VANETs," Future Generation Computer System, 2018.

[3] M. K. Hossain, S. Datta, S. I. Hossain, and J. Edmonds, "ResVMAC: A Novel Medium Access Control Protocol for Vehicular Ad hoc Networks," Procedia Computer Science, vol. 109, pp. 432-439, 2017.

[4] X. Jiang and D. H. C. Du, "PTMAC: A prediction-based TDMA MAC protocol for reducing packet collisions in VANET," IEEE Transactions Vehicular Technology, vol. 65, no. 11, pp. 9209-9223, 2016.

[5] T. L. Sheu and Y. H. Lin, "A cluster-based TDMA system for intervehicle communications," Journal of Information Science \& Engineering, vol. 30, no. 1, pp. 213-231, 2014.
[6] L. Zhang, Z. Liu, R. Zou, J. Guo, and Y. Liu, "A scalable CSMA and self-organizing TDMA MAC for IEEE $802.11 \mathrm{p} / 1609 . \mathrm{x}$ in VANETs," Wireless Personal Communication, vol. 74, no. 4, pp. 1197-1212, 2014.

[7] H. Yu, Z. He, and K. Niu, "STDMA for vehicle-to-vehicle communication in a highway scenario ," 2013 5th IEEE International Symposium on Microwave, Antenna, Propagation and EMC Technology Wireless Communication(MAPE), no. 61171099, pp. 133$138,2013$.

[8] W. Guo, L. Huang, L. Chen, H. Xu, and J. Xie, "An adaptive collisionfree MAC protocol based on TDMA for inter-vehicular communication," 2012 International Conference on Wireless Communication \& Signal Processing (WCSP), 2012.

[9] H. A. Omar, W. Zhuang, and L. Li, "VeMAC: A TDMA-based MAC protocol for reliable broadcast in VANETs," IEEE Transaction Mobile Computing, vol. 12, no. 9, pp. 1724-1736, 2013.

[10] L. Miao, F. Ren, C. Lin and A. Luo, "A-ADHOC: An adaptive realtime dis- tributed Vehicular Ad-hoc Networks," International Conference on Communications and Networking (ChinaCOM), pp. 16, Xi'an, China, Aug. 2009.

[11] W. Guo, L. Huang, L. Chen, H. Xu, and C. Miao, "R-MAC: Riskaware dynamic MAC protocol for vehicular cooperative collision avoidance system," International Journal of Distributed Sensor Networks, April 2013

[12] Batth R.S., Gupta M., Mann K.S., Verma S., Malhotra A. (2020) Comparative Study of TDMA-Based MAC Protocols in VANET: A Mirror Review. In: Khanna A., Gupta D., Bhattacharyya S., Snasel V., Platos J., Hassanien A. (eds) International Conference on Innovative Computing and Communications. Advances in Intelligent Systems and Computing, vol 1059. Springer, Singapore

[13] Safdar Hussain Bouk, G. K., "Hybrid Adaptive Beaconing in VehicularAd Hoc Networks: A Survey," International Journal of Distributed Sensor Networks, 2015.

[14] R. Zou, Z. Liu, L. Zhang, and M. Kamil, "A near collision free reservation based MAC protocol for VANETs," IEEE Wireles Communication Networking Conference (WCNC), vol. 2, pp. 15381543,2014 